\title{
The Classed Nature of Queer Nightclubs in Shanghai
}

\author{
Xingyu Wang
}

\author{
University College London, London, WC1E 6BT, United Kingdom. Email: xingyu.wang.21@ucl.ac.uk
}

\begin{abstract}
This research proposal is inspired by one of the author's field trips to an LGBTQ+ friendly bar and the observation of its classed nature. This study aims to explore the classed nature of queer nightclubs in Shanghai, which are social venues for the stigmatized group to foster resilience against oppression and exclusion. It argues that such classed nature will undermine some members' access to and utilization of group-level resources, perpetuating class stratification both inside the LGBTQ+ community and in the larger society. The paper proposes three questions: (1) What are the class characteristics of queer nightclubs? (2) What is the impact of such classed nature of nightclubs on the queer participants in terms of their utilization of resources to benefit themselves? (3) What factors, in addition to class, might affect the utilization of spatial resources in the nightclubs? This study underscores that the level of resilience resource utilization by stigmatized groups merits scholars' attention because a low level of group-level resource utilization undermines the value of these resources and may widen in-group health inequality.
\end{abstract}

Keywords: Queer, nightlife, nightclub, community, resilience

\section{INTRODUCTION}

Despite the recent unfriendly political atmosphere towards LGBTQ+ communities in Shanghai, the city had been widely recognized by many websites and newspapers, including China Daily, The Independent, Utopia-Asia.com, New York Times, etc., as the 'gay capital 'in China for a long time (Bao, 2018) [1]. With neo-liberal economic and ideological neo-liberalization development in China, queer spaces appeared across the country more than ever, with Shanghai at the forefront of the trend. However, in recent years, daytime LGBTQ+ events, including art exhibitions, performances, film screenings, and even the Pride Parade, the most iconic queer activity worldwide, are fading out of the sight. On the contrary, more and more events are held at night, which intrigued me to join one of them, to observe the way of socialization in the community. The first site I chose was the Pearl, a bar that also serves as a theatre, located in the inner-city area of Shanghai, being famous for its gay tolerance. Here's an excerpt of my field notes.

"Around 1/3 of the guests are foreigners... People sit together with each other, with no matter what nationality, gender, or whatsoever... I cannot see any apparent LGBTQ+ signs from the decoration, but they have a drag queen to host the...performance... The menus are presented in 2 to 3 languages, and the decoration here tries to give a 'petit-bourgeois ambiance'... The way people dress up seems to be fashionable... Most of the guests occasionally smile as they chat. Only a few of them, like three in ten, are on their phones... I can see a gay couple making out on the dance floor."

While people are drinking and waiting for the performance to start, I notice that their class distinction, fashion, lifestyle, and sometimes sexuality could easily be observed. In other words, these traits are exposed in the setting, together with a prospering sense of cosmopolitanism and high capitalism. The ways people approached and demonstrated these traits were classed and gendered, raced and sexed, symbolized and recognized by each other. These guests belong to a very similar class.

The classed nature of this space brought me to the awareness that part of the community cannot know, use, or afford to get into this space; even those who get in can suffer from low participation in activities and low utilization of spatial resources to benefit themselves. The problem is exacerbated because, according to my field observation and interviews, nightclubs are the main physical venues where urban LGBTQ+ people socialize, expand social networks, and reach out to the community in a mundane manner, resulting from the lack of gathering at ordinary meeting places. 
The stigmatized nature of queer people further manifested the problem. Group members for whom the support system are inaccessible are more likely to suffer from mental health issues due to their stigmatized nature as they lack community-based resources to cope with their excess minority stress. Therefore, the paper argued that the classed nature (the fact that the space is classed) of queer social spaces like nightclubs merits scrutinization. It undermines the stigmatized group's access to and utilization of group-level resources, which may widen the in-group health inequality in the future.

\section{RESEARCH QUESTIONS}

This study will be endeavored to explore the classed nature of queer nightclubs in Shanghai. Three research questions are proposed in particular. (1). What are the class characteristics of queer nightclubs? (2). What is the impact of such classed nature of nightclubs on the queer participants in terms of their utilization of resources to benefit themselves. (3). What factors, in addition to class, might affect the utilization of spatial resources in the nightclubs?

\section{BACKGROUND AND RELATED RESEARCH}

In this section, the author will explain the importance of queer spaces like LGBTQ+ friendly bars as a spatial resource for people of this community to cultivate resilience against social stigma. Specifically, the author will explain how a nighttime queer space acts as a resilience recourse by providing a physical venue for meeting with similar others, expanding social networks to gain social support, and building a sense of connectedness to the community.

The social stigma around LGBTQ+ people is widely documented in literature and underlines the crucial importance of a healthy social network among the stigmatized group. The contemporary working definition of stigma is provided by Herek (2009) [2], referring to 'the negative regard, inferior status, and relative powerlessness that society collectively accords to people who possess a particular characteristic or belong to a particular group or category'. The perpetration of stigma creates the experience of stigma by individuals and groups that became the "targets," which leads to adverse mental health impact. According to the meta-analysis by Meyer (2003) [3], gays and lesbians are about twice as likely as heterosexual people to experience mental health disorders, including substance use disorders, affective disorders, and suicide. However, queer social spaces ameliorate excess stress experienced by the stigmatized group, providing members the opportunity to develop social relations, interaction with other community members, and form a sense of community from group affiliations.
In light of the difficulty faced by the LGBTQ+ community scholars have made continued efforts to investigate the resilience of stigmatized people against excess stress that lessen the negative impact of stigma on their health and well-being. Queer spaces such as nightclubs provide a venue for queer people to meet similar others, which helps them develop a sense of self identity and improve their well-being. Drawing upon social identity and self-categorization theories, as well as social comparison and symbolic interaction theories, Meyer (2003) [3] pointed out the significance of interactions with people in developing a sense of self. Specifically, the self-categorization process provides an anchor for group and self-definition (Tajfel \& Turner, 1986; Turner, 1999) [4-5]. People gather meanings to their world and experiences from the social environments around them (Stryker \& Statham, 1985) [6]. This is rather important considering that in China, queer people are rarely able to gather in ordinary settings, while nightclubs provide such merit.

Moreover, nightclubs act as venues for bondbuilding and harvesting social support, which is another source of reliance for the stigmatized. Cohen (2004) [7] highlighted the role of social relationships in improving health conditions through two mechanisms: promoting social connectedness and stress-buffering. Upon the findings, Kwon (2013) [8] developed a theoretical framework of resilience in lesbian, gay, and bisexual individuals. Social support, emotional openness, hope, and optimism form the main sources of resilience. LGB people can benefit from better social support, more open emotional expressions, and more hope through lowered reactivity to prejudice and increased life satisfaction. In particular, the social support aspect can be reinforced by building connections with other community members, which is one of the crucial functions of queer social space like the Pearl. Specifically, as Cohen (2004) [7] reviewed, the social network helps people develop positive appraisals of stressful events. Social support is even more beneficial to LGB people from people who know their sexualities (Grossman, D’Augelli, \& Hershberger, 2000) [9]. Such acknowledgement by others may arise from one's appearance or the mere fact of attendance in a gay club or other queer spaces. In summary, support from other community members helps queer people to thrive and thus improve their resilience in coping with discriminatory events in life.

A sense of belonging and connectedness acts as another factor of resilience for people who are stigmatized. Meyer (2003) [3] reviewed that group solidarity and cohesiveness within minority groups protects them from health problems resulting from minority stress. Empirical studies confirm this assertion. For example, Branscombe, Schmitt, and Harvey (1999) [10] found that reinforced in-group identity is directly related to positive well-being in their study testing the 
effect of prejudice towards Black participants. In another study, Postmes and Branscombe (2002) [11] found that greater in-group acceptance in Black groups can lead to improved life satisfaction and well-being. According to Jones et al. (1985) [12], group affiliations provide the stigmatized people with two mechanisms for coping with minority stress: to place them in environments in which they are not stigmatized, and to offer them direct support. Group affiliations further construct the sense of belonging of individuals. According to Thoits (1985) [13], in such groups settings, the stigmatized individuals will conduct a reappraisal to compare themselves with other group members that are similar to them, which is helpful for mitigating harm to their mental health.

Personal coping strategies are crucial for stigmatized individuals to experience less psychological stress. However, it is group-level resources that decide the boundaries of individual coping efforts (Meyer, 2003) [3]. This interpretation highlights the extent to which an individual can access and utilize group resources to adopt self-enhancing values, which is much more variable in personal coping strategies.

Thus, inclusive queer spaces like queer nightclubs play an essential role in providing a group-level spatial resource where LGBTQ+ communities are free of marginalization and discrimination and can embrace their identities and social preferences. Therefore, the classed nature of such spaces is of great value to be scrutinized.

\section{METHODS}

The research aims to gather abundant and meaningful data about the nightlife of queer people in Shanghai and find clues indicating the extent of classed nature in the nightclubs. The research topic comes from the author's observation, but it is hypothesized that factors other than class can affect people's utilization of resources in the space. Finding out these factors is another aim of the study. The research will be centered around an inner-city bar in Shanghai, namely the Elevator, a famous queer bar in Shanghai, where I have been three times for a total of over 12 hours. The researcher, as well as this author, will try to find a job to stay there for a year, which would be the time length of data gathering. It is expected the observed pattern here can be generalized to other queer bars in the inner-city area of Shanghai.

In general, the methods involve participant observation, unstructured and semi-structured interviews, and structured questionnaires. The informal unstructured and semi-structured interviews are designed to resemble daily chat, while in-depth formal semi-structured interviews are also required to gather information about subjects' lifestyles in general.
Questionnaires will be spread online in the name of a questionnaire for customers satisfaction survey.

Socioeconomic status (SES) will be used to measure subjects' social classes, which takes income, education, and occupation into account in judging one's social class. Specifically, income refers to any flow of earnings received, including wages, salaries, profits, etc. Occupation is coded by the corresponding index of occupational prestige provided most recently by the American National Opinion Research Center. Once the occupation is known, one-thousandth of the average income of the job is added as a part of the SES. The valuation of the average income of each job is based on information provided by Knoema. Education is also taken into consideration because, according to American Psychological Association, better education is associated with higher income, more control, and broader social networking. The coding of education is based on the UNESCO 2011 international standard classification of education, which divides educational level into eight levels, with zero for illiterate and seven for a Ph.D. or equivalent. Information on these three aspects (income, education, and occupation) will be gathered mainly through informal chats and consumer surveys. A final SES score will be given to each subject to indicate the class to which they belong. To sum up, the equation this study will use to calculate SES is:

SES = Score for education level + Occupational prestige + Average income of the job/1000 (in RMB)

Fieldworks include taking field notes from participant observation and chat-like informal interviews with the customers. The observation and informal interviews will be carried out while I'm working in the bar. The observation aims to roughly determine the customers' social class as mentioned before, understand how they connect to the community and experience the sense of belonging, how they expand and utilize their social network, how they demonstrate their identity, what helps in the aforementioned processes, and finally, what improves the results. The main focus of the observation would therefore be the appearances and behaviors of the customers. More specifically, these are what would be noted in the observation:

1. The appearance - whether they are wearing brandy clothes and bags, whether they have expensive accessories. For regular visitors, whether they change the look frequently and maintain the level of spending on outlook may also be considered.

\section{The behaviors}

1. Whether and how they are interacting with others and making meaningful socializations. A simple greeting and eye contact are not considered meaningful socializations. 
2. Whether and how they are expanding their social network by meeting with strangers.

3. Whether and how they are expressing their identity. Examples can include wearing dramatic makeups, voguing (a type of dancing) to the music, acting intimately (e.g., kissing) with same-sex partners.

4. Whether and how they are engaged in collective behaviors such as group singing.

5. Whether and how they are engaged in other forms of utilization of resources from the space -- for example, if they are reacting to the music, drinking wines from the bar, interacting with bartenders and performers, photographing or videoing, and posing them online.

\section{Others}

6. What kind of music is preferred.

7. Nationalities of the customers.

8. If customers are comfortable with English usage.

9. If they come alone, come with fixed partners, or with different people all the time.

Each subject's appearance will be recorded, which is taken as a piece of information for the judgment of their class and as a way of differentiating them from each other. Example notes can be: an Asian male in a suit, Prada shoes, a fancy watch, or a Christian male in a black polo shirt with white jeans. Comprehensive information about people's appearance, helpful or not, will all be taken. However, people may be given a number for convenience. For instance, the Asian man in the suit may be coded as 21072411, indicating that he is the 11th subject in the field observation/interview carried on July 24th, 2021.

During the observation, the researcher will try the best to record as much information as possible. However, jottings are allowed when time is limited or too much information has to be taken. Music will be recorded if it's difficult to describe. Photographs can be taken with the permission of subjects. Informal chattype conversations will be conducted if the researcher wants to know more about any participant. The interview will be unstructured or semi-structured, but aiming to find out the following information:

1. How they get to know about the bar;

2. How they feel in the bar;

3. How often they come to the bar;

4. If they find the prices of wine too high;

5. If they are comfortable with conversing in English;

6. Personal background such as jobs;
7. Their social network;

8. If they find nightlife an important part of their socialization and why;

9. If they will come back to the place again;

10. If they go to other sites for socialization;

11. Their purpose of being here;

12.Their age.

With these in mind, example (grand tour) questions can include:

1. Are you still a student?

2. Did you come with your friends?

3. Do you like the music?

4. Are you new here?

5. Do you like the bar? How did you know this place?

\section{Want another drink?}

7.Have you been here yesterday? I found you quite familiar. How often do you come?

However, filler questions are also needed to guide the direction of the conversation. Friendly greetings and compliments can be used to build rapport, which would be very important. Conversations can be cut if the subject is reluctant to chat.

Along with the observations and informal chats, questionnaires will be spread to customers in the name of a customer satisfaction survey when they are still in the bar, so this researcher could know who the information is from. The purpose of this questionnaire is in accordance with that of the observation and the interview, but it will serve more as an auxiliary reference due to its shortcomings. For example, participants' answers can be biased by social disability, so they may lie about their income and jobs. Moreover, it is possible that data gathered are not enough to conclude a pattern that can be generalized to all customers because people are usually reluctant to disclose their personal information. However, the questionnaire can probably gather information about where else LGBTQ+ people go for socializing, which would indicate whether a bar is an appropriate field for the research.

Personal semi-structured interviews may also be carried outside of the research. The purpose of these interviews is to find if the interviewee feels connected to the community when they are within a queer bar and the origin of such connectedness. Moreover, if the information gathered in the field is not enough to conclude a pattern, questions related can also be asked as a supplement to the fieldwork. The interviewees are not limited to customers. In fact, the performers and 
staff will be the main group of interviewees.

Data collected are then organized, coded, and analyzed to interpret customers 'behavior. Gathered target information will be put into categories like:

A. The utilization of the space: interaction with others

B. The utilization of the space: expression of identities

C. The utilization of the space: expanding social networks

D. The utilization of the space: engagement in collective activities

E. Familiarity of the space (e.g., being a regular visitor, knowing the workers well, etc.)

F. Proof that nightlife is important for socialization

G. Other access to the community

H. All other information (e.g., bilingual, regular visitor, friends working here, etc.)

After organization and categorization, the data will be interpreted to answer the research questions.

\section{POSITIONALITY}

As somebody working in the bar, I can be a registrar at the front desk, a party warmer, a backstage assistant, or other roles. It is expected that my job at the venue will help the rapport building between me and the subjects, especially with those I will work with. Moreover, my knowledge of the vernacular of queer culture and my understanding of their life experience in a heteronormative society may also help facilitate the formation of a sense of togetherness between us. The job also offers me an emic perspective that assembles that of the cultural members. However, I am not sure if the observation will be overt or covert in its nature. Covert observation eliminates effects brought by my role as a researcher, but ethical questions may be raised. A compromising plan is that my colleagues will be informed of my position as a researcher but the customers will not.

It is also important to note that my position as a novice ethnographer alongside my cultural and social upbringing has largely shaped the methodological approach. Therefore, I need to observe and interview mindfully and resist the perspectives like male, Asian, or straight gaze, etc., embodied in the positionality.

\section{Preliminary findings}

It is expected that we can find significant clues indicating the classed nature of nightclubs in Shanghai. Meanwhile, people with higher SES scores can utilize resources better to benefit themselves in general. Moreover, in the process, factors other than class, such as social network, or personal reputation within the community, are also expected to impact the utilization of resources. However, the mechanisms are unknown at the moment.

More specifically, people with higher SES scores are expected to have more labels of A to E. Upper-class people may also showcase more confidence when interacting with strangers and find it easier to expand their social circles. They are usually the ones who implicitly and explicitly lead the action of the group they stay in. For instance, they may suggest a drink when the group sits at a bar or determine the time to leave. Moreover, they may initiate more collective actions than others, such as applauding the performers. It is also expected that they will be found to be conformable with English talking, more familiar with the environment, more likely to have companies, and more likely to be regular visitors to the space. However, it is unclear if people with higher class-standing will have a better reputation within the community. With the rise of cyber celebrity business, people who have more online followers are more likely to be known and respected. This phenomenon leads to the hypothesis that factors other than class, such as online popularity, can affect the utilization of spatial resources in queer nightclubs as well.

\section{CONCLUSION}

The study views nightclubs as crucial social venues for the stigmatized group to gain resilience against oppression and exclusion by getting access to the community, building a sense of connectedness, and expanding members' social networks. However, the classed nature of such spaces will undermine some members's access to and the utilization of group-level resources, perpetuating class stratification both inside the LGBTQ+ community and in the bigger society. It is expected that the findings of this study can offer insights for policymakers. For instance, the government may recognize the merits of nightclubs as resilience sources that are vital to the LGBTQ+ communities. The study's findings may also justify the importance of legal recognition and protection of the rights and interests of minorities so that they are free and safe to present their identities in the daytime.

The study may also offer new insights into urban spaces as venues for the oppressed to socialize. There have been many scholars investigating city spaces for queer consumptions. For example, Bell and Binnie (2004) [14] argued that urban queer spaces are highly commodified in the tournament of urban entrepreneurialism. However, there has been a research gap regarding what affects the utilization of resources inside these spaces. Therefore, the study is endeavored 
to investigate the way visitors use spatial resources to benefit themselves, arguing that the varying ability to take advantage of group-level resources is also crucial for the study of urban spaces.

Moreover, it is crucial for future researchers to bear in mind that sexual orientation is only one identity of LGBTQ+ people. Though sexual orientation and gender performance have tremendous impacts on many aspects of one's life, the interaction of this and other identities also merits consideration. Sometimes excessive attention is paid to a salient label, while influences of other identities, such as socioeconomic background, are overlooked.

In addition, the strictly limited generalizability of the findings is a challenge faced by cultural studies across different regions due to unequal levels of development. For instance, the results of this research project probably cannot be generalized to other cities considering that Shanghai is one of the most modern cities in China. Inclusive gay spaces are almost invisible in smaller cities, not to mention the rural areas. Thus, the sample of field sites is limited, and so is the applicability of the findings. Therefore, scholars need to notice nuanced contextual conditions at different regions when conducting culture-related studies, especially when the culture differs from the mainstream.

\section{REFERENCES}

[1] Bao, H.W. (2018), Chapter 2 Imagined Cosmopolitanism: Queer Spaces in Shanghai, Queer Comrades: Gay Identity and Tongzhi Activism in Postsocialist China. Marston Book Services Limited, Oxfordshire. Page 37.

[2] Herek, G. M. (2009). Felt stigma towards homosexuals measure. PsycTESTS Dataset. https://doi.org/10.1037/t14548-000.

[3] Meyer, I. H. (2003). Prejudice, social stress, and mental health in lesbian, gay, and bisexual populations: Conceptual issues and research evidence. Psychological Bulletin, 129(5), 674-697.

[4] Tajfel, H., \& Turner, J. C. (2004). The social identity theory of Intergroup Behavior. Political Psychology, 276-293.

[5] Turner, J. C. (1999). Some current issues in research on social identity and self-categorization theories. In N. Ellemers, R. Spears, \& B. Doosje (Eds.), Social identity: Context, commitment, content (pp. 6-34). Oxford, England: Blackwell.

[6] Stryker, S., \& Statham, A. (1985). Symbolic interaction and role theory. In G. Lindzey \& E. Aronson (Eds.), Handbook of social psychology (pp. 311-378). New York: Random House.
[7] Cohen, S. (2004). Social relationships and health. American Psychologist, 59(8), 676-684. https://doi.org/10.1037/0003-066x.59.8.676

[8] Kwon, P. (2013). Resilience in lesbian, gay, and bisexual individuals. Personality and Social Psychology Review, 17(4), 371-383. https://doi.org/10.1177/1088868313490248

[9] Grossman, A. H., D'Augelli, A. R., \& Hershberger, S. L. (2000). Social support networks of lesbian, gay, and bisexual adults 60 years of age and older. Journals of Gerontology: Psychological Sciences, 55B, P171-P179.

[10] Branscombe, N. R., Schmitt, M. T., \& Harvey, R. D. (1999). Perceiving pervasive discrimination among African Americans: Implications for group identification and well-being. Journal of Personality and Social Psychology, 77(1), 135149.

[11] Postmes, T., \& Branscombe, N. R. (2002). Influence of LONG-TERM Racial environmental composition on subjective well-being in African Americans. Journal of Personality and Social Psychology, 83(3), 735-751.

[12] Hilbert, R. A., Jones, E. E., Farina, A., Hastorf, A. H., Markus, H., Miller, D. T., \& Scott, R. A. (1985). Social stigma: The psychology of marked relationships. Contemporary Sociology, 14(3), 401.

[13] Thoits, P. A. (1985). Self-Labeling processes in mental Illness: The role of Emotional deviance. American Journal of Sociology, 91(2), 221-249.

[14] Bell, D., \& Binnie, J. (2004). Authenticating queer space: Citizenship, urbanism and governance. Urban Studies, 41(9), 1807-1820. 